See discussions, stats, and author profiles for this publication at: https://www.researchgate.net/publication/276061923

\title{
The Value of Past and Present Cues: An Evolutionary Perspective
}

Article in European Journal of Personality · January 2015

CITATIONS

0
READS

21 


\title{
The Value of Past and Present Cues: An Evolutionary Perspective
}

\author{
Felix D. Schönbrodt \\ Ludwig-Maximilians-Universität München, Germany
}

Birk Hagemeyer

Friedrich-Schiller-Universität Jena, Germany

In press at European Journal of Personality

\begin{abstract}
Author Note
Felix D. Schönbrodt, Department of Psychology, Ludwig-Maximilians-Universität München, Germany; Birk Hagemeyer, Friedrich-Schiller-Universität, Jena, Germany.
\end{abstract}

We want to thank Michael Zehetleitner and Marcus Mund for comments on our commentary.

Correspondence concerning this comment should be addressed to Felix Schönbrodt, Leopoldstr. 13, 80802 München, Germany. Email: felix@nicebread.de.

Phone: +4989 2180 5217. Fax: +498921803000. 


\begin{abstract}
In their target article, Rauthmann et al. (this issue) argue that situation research would be best off focusing on perceptions of situation, not on objective cues. In contrast, we advocate the importance of environmental cues by taking an evolutionary standpoint. From an ultimate perspective, cues from the phylogenetic past shaped our perceptual system and can guide the development of a theoretically informed structure of psychological situations. From a proximate perspective, cues are essential factors (beyond conscious perceptions) for the explanation of actual behavior, in particular when implicit processes are considered.
\end{abstract}




\section{The Value of Past and Present Cues: An Evolutionary Perspective}

Developing a coherent framework for research on situations is a Herculean task, and we commend the authors Rauthmann, Sherman, and Funder (this issue; henceforward called RSF) for taking it on. Considering the historical tension between "situationists" and "personality psychologists" it may look paradoxical, but it seems that personality psychologists have the greater inclination as well as the appropriate statistical tools to uncover the psychological structure of situations. This is all the more remarkable as there is an important difference between persons and situations. Whereas a person can be clearly defined as an entity in time and space, when/where a situation starts and ends is ambiguous. It seems safe to say that situations take place outside the boundaries of a person and are somehow limited in time and space, but consensus beyond that will be difficult to reach. Therefore, we circumvent the concept of "situations" and simply refer to the environment of an organism. We focus on the benefits of objectively definable components of the environment, or "cues", as termed by RSF. Specifically, we highlight (i) how cues from the phylogenetic past shaped our perceptual system and can guide the development of a theoretically informed structure of psychological situations, and (ii) why present cues are essential for the explanation of actual behavior, especially regarding implicit processes.

\section{Cues in the Past: The Ultimate Perspective}

Human perception can be expected to be tuned to those species-specific aspects of the environment that had consequences for genetic fitness in phylogenetic history. This link between the perceptual system and fitness-relevant aspects of the environment, however, does not imply 
that evolution shaped perception towards veridicality (i.e., to exact correspondence of perception and environment). Selection pressures can work in such a way that representations of situations (i.e., "characteristics" in RSF) are more adaptive when they are biased (Bischof, 2014; Haselton \& Buss, 2000; Hoffman, 2009; Zehetleitner \& Schönbrodt, 2015).

This ultimate perspective allows to analyze the structure of situation perception based on its adaptive value and has several implications. First, adaptive misrepresentations underscore the importance of those characteristics that systematically deviate from objective cues. Second, on the other hand, the ultimate perspective emphasizes an objectivist view on the environment. Psychological processing mediates the chain between cue and behavior, but in the end it is the objective cue that is fitness-relevant ${ }^{1}$ : The proof of the nutritional value is in the objective pudding, not in its perception (which can be misguided by artificial sweeteners). Third, the ultimate perspective suggests that universal as well as differential perceptions of cues have evolved to solve adaptive problems. We agree with RSF that situational taxonomies should be tailored to our "research needs" (p. 25) and add that such systems should be grounded in theoretical considerations informed by an evolutionary perspective (e.g., Penke, Denissen, \& Miller, 2007).

\section{Cues in the Present: What About Implicit Processing?}

The role of cues in the present refers to the proximate level of behavior causation and thus to the core business of psychologists. RSF's Processing Principle states that environmental information must be first processed, either explicitly (consciously) or implicitly (non-consciously), to become

1 We acknowledge that in sexual selection socially constructed characteristics that are not based on directly fitness-relevant cues could get relevant (cf. "Fisherian runaway process"). It is unclear, however, to what extent these seemingly superfluous ornaments typically are correlated with actual fitness benefits (Prokop, Michalczyk, Drobniak, Herdegen, \& Radwan, 2012). 
relevant for behavior. Furthermore, the Circularity Principle notes that perceptions of situations should be clearly separated from personal states or reactions.

On a conceptual level, we agree with both principles. However, in the elaborations of the two principles, especially regarding their practical application, we missed a consequent incorporation of implicit processes. There is a crucial difference between the assessment of explicit and implicit perceptions. The latter can only be indirectly assessed via reactions of the organism to environmental cues. Thus, at the operational level, implicit perceptions are inextricably tied to personal states. This violates the Circularity Principle. To avoid such violations, research on implicit processes must use cues rather than characteristics. This approach, however, does not fit well to the Processing Principle, which "alerts us that we should attend to people's experiences" (p. 17). Hence, the Processing Principle and the Circularity Principle combined seem to confine (or at least to align) the proposed framework to consciously accessible perceptions of situations. This, however, might only touch the tip of the iceberg of environmental influences. To untangle the consensual stratum (i.e., the shared perception of several perceivers) from the idiosyncratic stratum (i.e., the unique processing of a single perceiver), RSF suggest variance decomposition via Social Relation Models (SRM). We agree that SRM can be an appropriate tool for situation research. However, in virtually all practical applications, the SRM has only been used with explicit ratings. A notable exception is reported in Krause, Back, Egloff, and Schmukle (2014), who were the first to apply the SRM to an implicit measure of liking. This exciting new approach allows the separation of consensual from idiosyncratic reactions at the implicit level, but does not resolve the confound of personal reactions with environmental perceptions. For a clear distinction between person and environment, cues are important complements that deserve 
a more prominent place in a psychology of situations, in particular with regard to implicit processes.

\section{Conclusion}

RSF proposed that "research will be best off focusing on perceptions of situation characteristics" and that this kind of situation research will be the "most productive" (p. 17). While we agree that the processed perceptions are important, we advocate the relevance of cues. First, objective environmental features constituted the selection pressure that shaped our senses and categories. Therefore, we are convinced that a search for the psychological structure of situations will benefit from an evolutionary background. Second, at least at the operational level, the proposed framework confines characteristics to consciously processed and reportable perceptions of situations. But whenever implicit processes operate, cues are essential for a clear distinction between person and environment.

\section{References}

Bischof, N. (2014). Psychologie: Ein Grundkurs für Anspruchsvolle (3. edition). Stuttgart: Kohlhammer.

Haselton, M. G., \& Buss, D. M. (2000). Error management theory: A new perspective on biases in cross-sex mind reading. Journal of Personality and Social Psychology, 78, 81-91. doi:10.1037/0022-3514.78.1.81 
Hoffman, D. (2009). The interface theory of perception: Natural selection drives true perception to swift extinction. In S. Dickinson, M. Tarr, A. Leonardis, \& B. Schiele (Eds.), Object categorization: Computer and human vision perspectives (pp. 148-165). Cambridge, UK: Cambridge University Press.

Krause, S., Back, M. D., Egloff, B., \& Schmukle, S. C. (2014). Implicit interpersonal attraction in small groups: Automatically activated evaluations predict actual behavior toward social partners. Social Psychological and Personality Science, 5, 671-679. doi:10.1177/1948550613517723

Penke, L., Denissen, J. J. A., \& Miller, G. F. (2007). The evolutionary genetics of personality. European Journal of Personality, 21, 549-587. doi:10.1002/per.629

Prokop, Z. M., Michalczyk, Ł., Drobniak, S. M., Herdegen, M., \& Radwan, J. (2012). Metaanalysis suggests choosy females get sexy sons more than "good genes." Evolution, 66, 26652673. doi:10.1111/j.1558-5646.2012.01654.x

Zehetleitner, M., \& Schönbrodt, F. D. (2015). When misrepresentation is successful. In T. Breyer (Ed.), Epistemological dimensions of evolutionary psychology (pp. 197-222). New York: Springer. 